\title{
APPLICATIONS OF INDIRECT ACCRETION DISC IMAG- ING TO ORBITAL SOLUTIONS FOR PRIMARY STARS
}

\author{
MARTIN STILL ${ }^{1,2}$ \\ 1. University of St. Andrews, Physics \& Astronomy, North \\ Haugh, St. Andrews, Fife KY16 9SS, UK \\ 2. Astronomy Centre, University of Sussex, Falmer, Brighton \\ BN1 9QH, UK
}

The general inability of optical observations of low mass interacting binaries to provide accurate radial velocity measures for the stellar components is a problem for which there is no universally applicable solution. Velocity measurements are handicapped because the accretion disc about the primary often dominates the optical spectrum. Therefore spectroscopists resort to measuring line profile symmetry in individual spectra of the accretion disc in order to provide information on the primary's motion. However, discs are not axisymmetric structures and the standard approach generally fails because the asymmetry-free inner disc emission is usually dominated by statistical noise.

We adopt an algorithm which tests for axisymmetry in tomograms. This approach is an improvement on the standard method because (i) the form of symmetry search has changed to include the whole data set, and (ii) asymmetries are removed, providing they are not too dominant, by median averaging annuli about test velocities. Primary velocities are given by the minima in velocity maps of a summed residual distribution which is the result of removing the computed symmetric profile from the data.

In Fig. 1 two test cases for the algorithm are plotted. The first is a model of optically thin line emission from a disc and bright spot, about a primary with a projected radial velocity semi-amplitude of $K=100 \mathrm{~km} \mathrm{~s}^{-1}$. The standard method provides a 'best' solution of $K=122 \pm 3 \mathrm{~km} \mathrm{~s}^{-1}$, where the phase lag between systemic velocity crossing and primary superior conjunction is $\phi_{0}=18^{\circ} \pm 3^{\circ}$. Clearly this is unacceptable. However, the minimum in the residual distribution presented in Fig. 2 provides an orbital solution of improved accuracy: $K=97 \pm 6 \mathrm{~km} \mathrm{~s}^{-1}$ and $\phi_{0}=0.3^{\circ} \pm 0.1^{\circ}$. The second case concerns Balmer emission from the non-eclipsing quiescent dwarf nova LY Hya. The standard approach provides $K=133 \pm 4 \mathrm{~km} \mathrm{~s}^{-1}$. However the phasing of this solution indicates that the bright spot location is not 


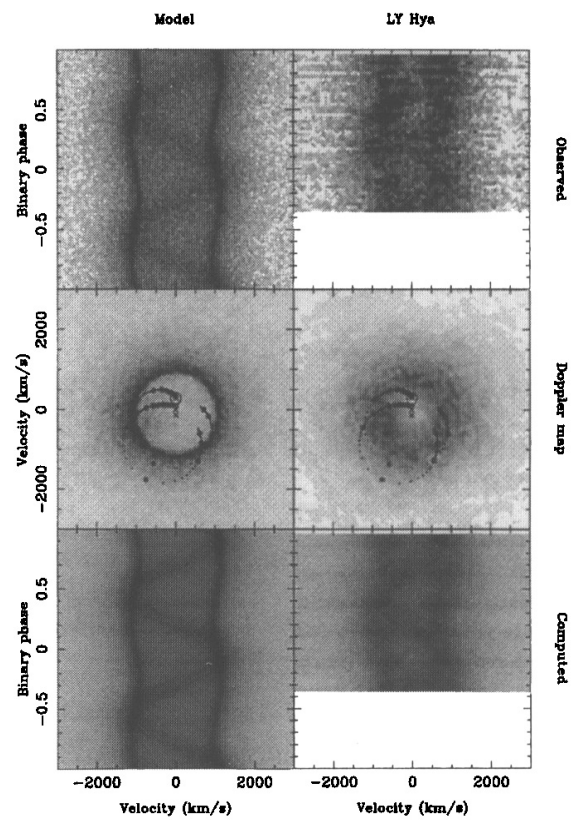

Figure 1. Data and tomograms of our two test cases - optically thin line emission from a disc and bright spot and Balmer emission from the quiescent dwarf nova LY Hya.

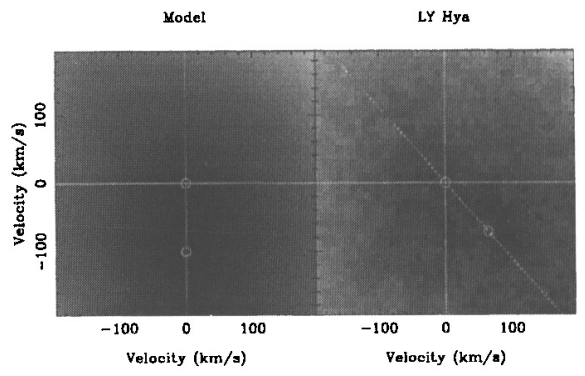

Figure 2. Velocity maps of the summed residual after removing axisymmetric emission from the line profile. The minima in these distributions predict the primary velocity, circles represent centres of mass and predicted primary velocities.

compatible with flow off the canonical gas stream trajectory (Fig. 1). Either the solution is inaccurate or a physical model is required to explain the peculiar bright spot distribution. Our algorithm provides $K=97 \pm 31 \mathrm{~km} \mathrm{~s}^{-1}$, suggesting that the previous determination was biased. More significantly, the phasing has been redetermined (dashed line, Fig. 2), providing a more canonical location for the bright spot emission. 